\title{
HIGHLIGHTS
}

\section{Multitasking for base editors}

ce

a range of
potential
applications
involving

more complex

compound

edits than are

achievable by

single base

editors

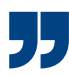

DNA base editors achieve genome editing without requiring DNA double-strand breaks as part of their mechanism. However, the range of possible edits is limited because base editors are typically either cytosine base editors (CBEs; for $\mathrm{C} \rightarrow \mathrm{T}$ edits) or adenine base editors (ABEs; for $\mathrm{A} \rightarrow \mathrm{G}$ edits). Three new studies report dual base editor systems for combinatorial editing in human cells.

Existing DNA base editor systems generally involve a single-strand nickase variant of Cas9 (Cas9n) fused to one type of deaminase. For CBEs, the deaminase is CDA1, AID or an APOBEC protein, and the fusion also contains one or two uracil glycosylase inhibitor (UGI) domains to inhibit excision of the intermediate uracil base. For ABEs, the deaminase comprises one or two TadA domains. The resultant fusion protein is directed to a target site by a guide RNA. All three new studies combine Cas9n with cytosine and adenine deaminases in a single fusion protein.

Grünewald et al. designed their dual base editor on the principle of combining the most promising single base editors, as well as economizing on the size of the construct to facilitate delivery and expression. They combined Target-AID (a CDA1-based CBE) and miniABEmax-V82G, both

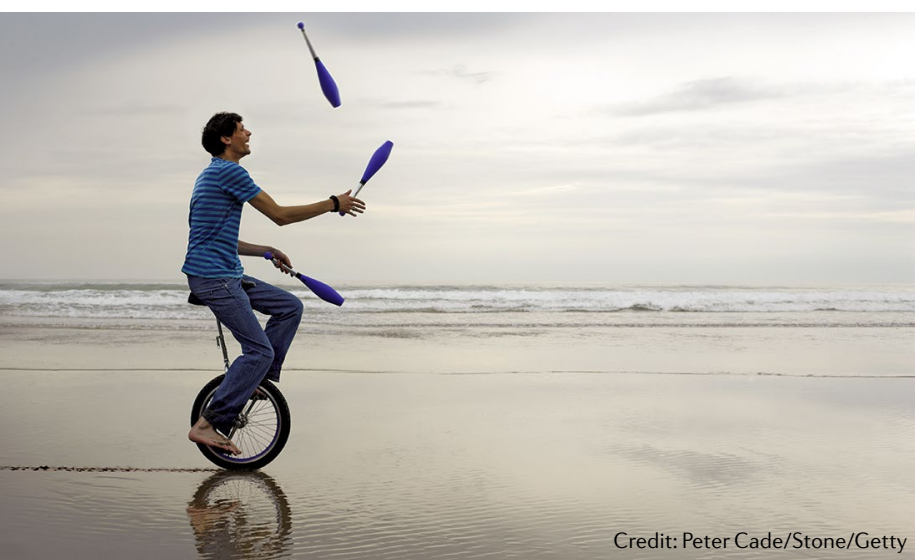

of which have among the lowest RNA off-target activity of existing base editors. Furthermore, 'max' refers to additional optimization such as for codon usage and nuclear localization sequence. The authors termed the resultant construct synchronous programmable adenine and cytosine editor (SPACE).

In human cells, SPACE retained the main desirable features of the constituent single base editors, such as the efficiency of generating the desired edits, as well as low rates of unintended insertions or deletions (indels) at the target site and infrequent off-target edits elsewhere in the genome and in RNA. Importantly, SPACE achieved more efficient dual editing of target sites than co-expressing the individual editors.

Zhang et al. tested various arrangements of ABE7.10 combined with different CBEs. The most efficient construct combined human AID with ABE7.10, and subsequent further optimizations to the sequence resulted in their A\&C-BEmax construct.

Relative to comparable single editors, A\&C-BEmax had slightly reduced activity towards adenine but increased activity towards cytosine in human cells, and it displayed similarly low indel frequency and off-target effects on DNA and RNA.

As proof of principle for therapeutic gene editing, Zhang et al. sought to accomplish a double edit in the regulatory sequences of the $H B G 1$ $\gamma$-globin gene, for which upregulation is a potential treatment for sickle cell disease and $\beta$-thalassaemia. The required double edit could be achieved by A\&C-BEmax more efficiently than through co-expressed single base editors, and these edits successfully upregulated HBG in erythroid progenitor cells.

Sakata et al. generated and tested three different dual base editors:
Target-ACE combines Target-AID and ABE7.10, Target-ACEmax combines Target-AIDmax and ABEmax, and ACBEmax combines BE4max and ABEmax. Of these constructs, Target-ACEmax had the best dual editing performance, with similar on-target editing efficiencies relative to the constituent single editors.

For DNA off-target analysis, in contrast to the targeted sequencing of the other studies, Sakata et al. performed whole-exome sequencing and showed similarly low off-target DNA editing relative to the single editor constructs.

Importantly, the RNA sequencing analysis of Sakata et al. revealed a strikingly higher rate of unwanted RNA editing for the ACBEmax construct containing the APOBEC1 CBE from BE4max but low RNA editing in the other (CDA1-containing) constructs. This is consistent with recent reports of notable RNA off-target activity of some APOBEC-based CBEs and serves as useful external validation for the choice to use non-APOBEC CBEs in the studies of Grünewald et al. and Zhang et al.

These new dual base editors have a range of potential applications involving more complex compound edits than are achievable by single base editors, such as for therapeutic correction of disease alleles, or the generation of genetic diversity for genetic screens, lineage tracing or directed evolution.

Darren J. Burgess

ORIGINAL ARTICLES Grünewald, J. et al. A dual-deaminase CRISPR base editor enables concurrent adenine and cytosine editing. Nat. Biotechnol. https://doi.org/10.1038/s41587020-0535-y (2020)|Zhang, X. et al. Dual base editor catalyzes both cytosine and adenine base conversions in human cells. Nat. Biotechnol. https://doi.org/10.1038/s41587-020-0527-y (2020) |Sakata, R. C. et al. Base editors for simultaneous introduction of C-to-T and A-to-G mutations. Nat. Biotechnol. https://doi.org/ 10.1038/s41587-020-0509-0 (2020) RELATED ARTICLE Rees, H. A. \& Liu, D. R. Base editing: precision chemistry on the genome and transcriptome of living cells. Nat. Rev. Genet. 19 770-788 (2018) 